présure présentant à peu près la même flore microbienne. Les cultures en petit-lait de leur côté sont plus virulentes que les cultures sur recuite.

c) Influence de l'acidité des cultures ou présures.

On sait que les ferments lactiques finissent par périr sous l'influence de l'acide lactique qu'ils produisent. Avant de périr ils s'affaiblissent. L'expérience du laboratoire a démontré qu'à partir de 35 à $40^{\circ}$ d'acidité le pouvoir acidifiant des cultures diminuait progressivement à mesure que l'acidité augmentait. Les cultures les plus virulentes sont celles qui ont une acidité de 25 à $35^{\circ}$ SohxletHenkel au bout de 24 à 36 heures.

d) Influence de l'âge des cultures.

L'âge des cultures a sur leur pouvoir acidifiant un effet analogue à celui de l'acidité. La virulence des cultures augmente jusqu'à un certain moment et décroît ensuite avec l'âge. En général des cultures de 24 à 36 heures sont les plus actives.

Cet exposé des facteurs qui modifient la virulence ou le pouvoir acidifiant des présures ou cultures permettra peut-être au fromager de mieux adapter sa présure au lait de chaudière dont il dispose.

\title{
QUELQUES CONCLUSIONS PRATIQUES TIRÉES DE HUIT ANS D'ÉTUDE DE LA IMAMMITE EN EUROPE ET EN AMÉRIQUE DU NORD
}

par

\author{
J. M. ROSELL \\ Docteur en Médecine,
}

Chef du Département de Bactériologie,

Institut Agricole de l'Université de Montréal, Oka, Québec.

Bactériologiste, Ecole provinciale de Laiterie, Québec.

(Fin.)

\begin{abstract}
Valeur comparative au point de vue du diagnostic de chacun de ces tests chimiques et biologiques pour le diagnostic de la mammite. - De nombreuses et copieuses études ont été publiées sur ce sujet pendant les dix dernières années. Les résultats d'études complètes ont été publiés, en dehors de nombreuses autres publications, dans les ouvrages de BREED, BAKER, KLIMMER, Seelemann, Gloy, Bischoff, Rooder, Hucker, Miller et RoselL. Nous allons maintenant résumer les idées qui nous paraissent les plus acceptables après avoir étudié la question pendant près de huit ans.
\end{abstract}


Chacun de ces tests peut être suffisant pour donner un diagnostic net de la mammite quand les résultats obtenus expriment suffisamment clairement l'anormalité pathologique du lait. Les tests de la catalase et du chlore sont peut-être les plus sûrs pour le diagnostic ; viennent ensuite le $p H$ et le test du sédiment. On doit noter à cet égard que la catalase et le $p H$ n'ont une valeur qu'avec du lait frais ou des échantillons conservés en bon état. Les tests du chlore et du sédiment sont applicables à toutes les sortes de laits, même au lait caillé. Dans nos recherches comparatives sur environ 4.200 tests de laits différents, nous avons trouvé que les résultats obtenus étaient d'accord dans environ $85 \%$ des cas pour les tests du chlore, de la catalase et des leucocytes, et j'estime que tous cess tests, même pris isolément, constituent les moyens les plus convenables pour déceler la mammite. Le test du $p H$ exécuté à de nombreuses reprises sur le lait de quartiers séparés vient, à mon avis (lequel est analogue à celui de plusieurs autres expérimentateurs), en second, mais tandis que, sur certains troupeaux, il a été trouvé utilisable dans la proportion de 70 à $85 \%$ pour déceler la mammite, sur d'autres, chose surprenante, il n'a pas décelé la mammite dans un aussi grand nombre de cas.

Nous n'avons pas eu l'occasion d'observer de variations considérables dans le $p H$ d'un quartier à un autre, sur des vaches pour lesquelles les autre tests avaient aussi prouvé que le lait n'était pas anormal.

Si on désire déceler toutes les vaches d'un troupeau atteintes de mammite, nous conseillons la méthode suivante, que nous avons presque toujours adoptée : d'abord, examiner toutes les vaches au point de vue du $p H$, chaque quartier pris isolément, et ceci, si possible, deux ou trois heures avant l'heure habituelle de la traite. 60 à $85 \%$ des quartiers atteints peuvent être ainsi décelés, d'après notre propre expérience, à la vitesse de 20 vaches à l'heure. Pratiquer, en même temps, l'épreuve de la catalase rapide, en ajoutant 1 à 2 cc. d'eau oxigénée à 6 ou $9 \%$, dans le lait utilisé pour J'épreuve au bleu de bromothymol. On peut encore faire l'épreuve rapide de chlore. Prélever des échantillons sur les quartiers restés négatifs ou douteux au $p H$, pour effectuer au laboratoire les tests du chlore, de la catalase, du sédiment, et bactériologique, et tous autres tests ; il est aussi recommandé de prélever des échantillons sur les quartiers anormaux trouvés positifs au $p H$ afin de les examiner aussi au point de vue du chlore, de la catalase, etc., si ces épreuves faites à l'étable ne suffisaient.

Pour examiner au laboratoire des échantillons de laits mélangés, les meilleurs tests à utiliser sont ceux du chlore et du sédiment, et 
peut-être aussi celui du lactose, et enfin si le lait n'est pas trop âgé, ceux de la catalase et du $p H$.

\section{TEST MACROSCOPIQUE DU SÉDIMENT}

Si on centrifuge $10 \mathrm{~cm}^{3}$ de lait propre et normal à 3.000 tours par minute pendant 15 minutes, on ne doit trouver qu'un très léger sédiment ou pas de sédiment du tout. Un sédiment dépassant environ $0 \mathrm{~cm}^{3} 1$ peut être considéré comme anormal, surtout si la couleur indique la présence de pus (jaune) ou de sang (rouge). Du lait propre et normal ne laisse pratiquement aucun sédiment dans un échantillon de $10 \mathrm{~cm}^{3}$ de lait.

\section{TEST DU TAMIS OU DU TISSU SOMBRE}

Dans les cas chroniques de mammite streptococcique, nous n'aurions découvert qu'un pourcentage relativement faible des quartiers atteints si nous n'avions employé que le test du tamis ou tissu sombre. Dans certaines formes de mammite, $6 \%$ seulement des vaches atteintes furent trouvées avec le test du tamis ou du tissu sombre, effectué comparativement avec les autres tests.

\section{TEST MICROSCOPIQUE DU SÉDIMENT}

Nous estimons, en étudiant les résultats de ce test, qu'une moyenne de 6 à 10 leucocytes par ehamp dans le sédiment non dilué est un chiffre normal maximum, 10 à 12 , ou plus, correspondant à des cas de maladie. Ce chiffre a été établi d'après les recherches de plusieurs autres chercheurs, et d'après les miennes propres. On pense, toutefois, que dans des quartiers parfaitement sains, la teneur en leucocytes du sédiment devraịt être moindre, ne devant pas vraisemblablement dépasser cinq à six cellules par champ. Si la préparation est faite avec du lait non sédimenté, mes travaux ont prouvé que, dans les quartiers sains, on trouve moins d'un leucocyte par champ de microscope ordinaire, ce qui fait moins de 500.000 leucocytes par centimètre cube. Nous croyons aussi, avec Húker et Trudell-Henning, qu'un lait contenant un nombre de cellules supérieur à 150.000 par centimètre cube doit être soupçonné de mammite.

En exécutant le test du sédiment, il doit être noté qu'il peut y avoir répartition irrégulière des leucocytes, en raison de la tendance qu'ont ces cellules à monter dans la couche de crème du lait, et par conséquent à être absentes dans le sédiment. Ceci peut être évité à un certain point en diluant le lait avec une quantité égale d'eau distillée et en chauffant le mélange à $45^{\circ} \mathrm{C}$. ou $50^{\circ} \mathrm{C}$. avant la centrifugation. En centrifugeant ce mélange, il est essentiel que la vitesse maximum de la centrifuge soit atteinte aussi rapidement que possible. 


\section{VARIATIONS DES QUANTITES COMPARATIVES DE LAIT DANS DES QUARTIERS DIFFERENTS}

De longues études comparatives ont montré que chaque quartier isolé de la mamelle donne, aussi longtemps qu'il est en condition normale, une quantité de lait constante et égale, qui ne change que suivant un degré physiologique limité, et qui est, jusqu'à un certain point, égal et proportionnel aux produits des autres quartiers.

La différence physiologique entre le lait de différents quartiers ne dépasse pas 200-300 $\mathrm{cm}^{3}$ pour une traite. Généralement, les quartiers postérieurs donnent plus de lait que les quartiers antérieurs correspondants. La différence semble être de 4 pour 5.

Des études spéciales sur des mamelles malades m'ont amené à conclure qu'une différence en moins de plus de 300 à $400 \mathrm{~cm}^{3}$ par quartier, si tous les quartiers sont traits en même temps, laisse soupçonner une mammite.

\section{BACTÉRIOLOGIE DE LA MAMMITE STREPTOCOCCIQUE}

D'après ma propre expérience, la méthode habituelle consistant à prélever des échantillons à l'étable afin de préparer des eultures bactériennes pour le diagnostic de la mammite, a souvent pour résultat la contamination de l'échantillon par la peau et les parties environnantes de la vache. Il est difficile, sur des échantillons envoyés à un laboratoire, d'affirmer qu'un microorganisme trouvé dans le lait provient réellement de l'intérieur de la mamelle. Comme Howard BRown l'indique dans une étude parue dans un récent numéro de The Cornell Veterinarian: « Les streptocoques ne sont pas un constituant nécessaire du lait, mais ils existent d'une façon inévitable dans tous les laits vendus. "

C'est pourquoi il est recommandé, en prélevant des échantillons pour une étude bactériologique, de ne tirer qu'un filet de lait dans un petit tube à essai stérile, après avoir lavé les flancs avec de l'eau et la mamelle avec de l'alcool (à $65 \%$ ) ou une autre solution antiseptique. Il faut aussi avoir fixé les queues des vaches voisines, jeter les premiers filets de lait, et éviter autant que possible la traite en atmosphère poussiéreuse.

Nos études nous amènent à accepter les conclusions suivantes :

La forme ordinaire de la mammite est pour ainsi dire exelusivement d'étiologie streptococciqua, d'autres formes étiologiques de mammite n'étant trouvées que très rarement. Ces dernières formes non streptococciques ont habituellement quelques aspects cliniques différents. La mammite staphylococcique, étant moins fréquente et de durée plus courte, est d'une importance économique moindre si on la compare à la mammite streptococcique, trouvée d'une façon certaine dans les troupeaux laitiers produisant du lait d'une façon intensive. 
Les streptocoques trouvés dans cette forme de mammite doivent être considérés dans la plupart des cas comme une variété des streptocoques. Ils ont quelques caractéristiques différentes qui les différencient des groupes de streptocoques humains décrits comme pyogenes, anginosus et epidemicus, et n'ont pas les caractéristiques de la plupart des types connus de streptocoques pathogènes d'animaux. Dans leur forme la plus commune, connue sous le nom de streptococcus mastitidis ou str. agalactia, ils ne sont pas bêta-hémolytiques. Ils produisent quelquefois une hémolyse partielle dans le sang de vache et le sang humain. Ils ne sont pas pathogènes pour les souris, les cochons d'Inde, les lapins, les chèvres, les porcs et les vaches, excepté quand ils sont inoculés à l'intérieur de la mamelle, car, dans ce cas, ils produisent ordinairement la mammite. On n'a jamais prouvé qu'ils soient pathogènes pour l'homme; toutefois, certains pensent qu'ils peuvent causer des troubles chez les enfants. Ces streptocoques présentent quelques caractéristiques culturales et des réactions biologiques qui sont plutôt constantes, et manifestent un manque d'action pathogène et toxique que quelques streptocoques hémolytiques semblent avoir. Ils semblent être similaires aux streptocoques décrits par NocARDMollereau (1884) sous le nom de streptococcus agalactia chronica contagiosa, par GUillebeau, Kitt, AdDamentz, ZozchkKe sous le nom de streptococcus mastitis epidemica indurativa, et enfin par Savage et Migula (1906) sous le nom de streptococcus mastitidis. Je suis d'avis que ce streptocoque est plus semblable au type lactique, spécialement au streptococcus cremoris, et au type streptococcus mitis, fecalis et salivarius, qu'aux autres types mieux connus de streptocoques.

Dans beaucoup de cas de mammite, je n'ai pas été eapable d'isoler le type ordinaire de streptocoques de la mammite dans des essais répétés, ni de les voir dans les sédiments étalés d'échantillons de laits frais ou incubés, prélevés aseptiquement sur des mamelles malades. Dans la plupart des eas, j'ai trouvé dans les étalements sédimentaires et dans les cultures des microcoques d'aspect aplati avec des caractéristiques culturales semblables aux microcoques saprophytes de la mamelle d'un blane transparent le premier jour, opaque les jours suivants. Dans mes premières études, je n'avais pas pensé que ces microcoques pouvaient avoir un rapport étiologique avec la maladie, mais après n'avoir constamment trouvé chez quelques vaches malades que des microcoques et ceux-ci en plus grand nombre que dans les échantillons de lait ordinaire, j'en arrive à penser, comme d'autres, que les microcoques saprophytes de la mamelle peuvent, dans des circonstances favorables, être un facteur étiologique de la mammite. J'ai trouvé que ce type, qui 
pouvait être dénommé mammite micrococcique, est de caractère plus bénin que la forme streptococeique.

Ces microcoques de la mamelle semblent très souvent mélangés avec les streptocoques et ne sont séparés des streptocoques en cultures pures qu'avec beaucoup de peine. Ce mélange très fréquent de streptocoques et de microcoques en culture supposée pure obtenue d'une mamelle mammiteuse, est la cause de variations fréquentes des caractéristiques culturales dans les sous-cultures répétées provenant de souches de mammite supposées pures.

Dans les formes de mammite chronique ordinaire ou sousclinique, qui, je erois, constituent $95 \%$ ou plus des mammites intéressant actuellement l'industrie laitière, on peut dire que pratiquement seuls les streptocoques sont obtenus en rapport étiologique avec la maladie.

Les aspects culturaux les plus importants de ce type central ou variété particulière de streptocoque peuvent être résumés comme suit: Aspect de la colonie de streptocoques sur agar nutritif ordinaire : ronde, bords légèrement granulés, lisse, transparente, avee un centre légèrement sombre, et généralement pas plus grande que $1 \mathrm{~mm}$. après 48 heures. Les colonies peuvent être plus petites $(0 \mathrm{~mm}, 5)$, mais sont rarement plus grandes que $2 \mathrm{~mm}$. Quelquefois, sur agar humide, le bord observé au microscope semble légèrement courbé et irrégulier. Quand on la touche avec une anse de platine, la colonie paraît avoir une consistance visqueuse. Les colonies n'ont pas toujours le même aspect lisse.

Sur bouillon séro-lactosé, $p H \quad 7,2$ à 7,4 , après 16 à 24 heures d'incubation, un dépôt floconneux se produit, laissant le liquide au-dessus clair et transparent. Il y a, toutefois, quelques cultures de streptocoques de la mammite qui, dans certaines eirconstances, troublent le bouillon, surtout. les streptocoques du type en chaînes courtes ou diplocoques.

Lait tournesolé, 7 à $10 \%$ de teinture de tournesol. Ce milieu est un des plus convenables pour différencier les streptocoques de la mammite des streptocoques lactiques, pyogènes et autres. Le streptocoque de la mammite en culture fraîchement préparée produit à $37^{\circ} \mathrm{C}$. les changements typiques suivants dans le lait tournesolé préparé avec la teinture de tournesol : d'abord, le milieu est acidifié et rougit en 18 à 24 heures; puis, le lait est coagulé en 20 à 24 heures, produisant un caillé consistant et homogène, avee ou sans apparition d'un peu de sérum. On voit souvent des stries se produire le long du caillé. $A u$ fond du tube de culture, apparaît souvent une légère réduction du tournesol. Cette réduction ne s'étend pas au delà de la demi-hauteur de la colonne de lait et, après 3 ou 4 jours d'incubation à $37^{\circ} \mathrm{C}$., la réduction est plus limitée si la colonne est haute (10 à 
$15 \mathrm{~cm}$.). Les streptocoques lactiques la ramènent d'abord à une couleur blanche avec un anneau rouge étroit restant au sommet du tube, puis ils coagulent le lait en 24 heures. Il est très rare que d'autres variétés de streptocoques produisent dans le lait tournesolé des modifications analogues à celles produites par les formes lactiques et les formes de la mammite. Si ceci a lieu, comme c'est quelquefois le cas avec le streptococcus pyogenes, les autres caractéristiques culturales permettent habituellement la différenciation. Quelques rares races de streptocoques de la mammite peuvent produire une réduction plus ou moins prononcée du lait tournesolé après deux ou plusieurs jours, et il y a aussi quelques races produisant la coagulation et l'acidification, mais seulement après 36 à 48 heures.

Lait au bleu de méthylène : $(1: 20.000)$. La majorité des races de streptococcus mastitidis ou agalactia ne produisent pas de changement dans ce milieu. Toutefois, W. T. MiLler a trouvé que la nature du colorant employé dans ce milieu a une influence considérable.

Agar-sang : Le streptococcus mastitidis produisant la réaction typique sur le lait tournesolé, sur le lait au bleu de méthylène, $p H$ final de 4,4, ayant une forte capacité de clivage sur l'hịppurate de sodium, faisant fermenter le sucrose, le lactose, et pas le raffinose, qui sont les caractéristiques les plus constantes de ce streptococcus, n'est jamais bêta-hémolytique.

$p$ H final: Dans le bouillon lactosé après 48 heures : 4,4 à 4,5 .

Clivage de l'hippurate de sodium : Une des caractéristiques du streptococcus mastitidis, qu'on ne trouve pas au même degré dans les autres streptocoques d'origine animale et encore moins dans ceux d'origine humaine, est sa forte capacité de clivage de l'hippurate de sodium.

Les sucres qu'il fait fermenter sont les suivants : lactose, sucrose, galactose, salicine, levulose, maltose, dextrine, amidon, trehalose.

Les sucres qu'il ne fait pas fermenter sont les suivants : mannite, inuline, raffinose, arabinose, xylose et sorbite. Quelques races ne font pas fermenter la salicine et très peu font fermenter le mannite. Les caractéristiques permettant la différenciation de ce streptocoque des autres sont les suivants : fermentation du lactose et du sucrose, non-fermentation du raffinose, et modifications caractéristiques du lait tournesolé, forte faculté de clivage de l'hippurate de sodium, pas de développement à $10^{\circ} \mathrm{C}$., $p$ H final de 4,4 , earactère non hémolytique, pas de pathogénité pour les animaux du laboratoire. S'il est injecté dans la mamelle d'un cochon d'Inde femelle en période de lactation, il produit une induration fibreuse après deux jours, durant deux semaines et plus. 
Dans une étude de la mammite, poursuivie, depuis quatre ans, à l'Ecole vétérinaire d'Oka (ROSELL, TRUDELL, BROCHU), sur environ 400 races isolées de quartiers séparés de mamelles malades; dans une autre étude (effectuée par MTLLer et Rosell, à la Division pathologique du Bureau d'Industrie animale de Washington), puis dans une étude comparative de 208 souches de streptocoques d'origine humaine et animale faite au Dép. de Bactériologie de l'Université de Me Gill (ROSELL-ShaRKeY), nous avons trouvé que les caractéristiques énumérées ei-dessus sont celles des streptocoques existant presque toujours dans les mamelles atteintes. Nous pouvons affirmer qu'environ $97 \%$ des streptocoques isolés par nous, avec les soins aseptiques les plus minutieux, des quartiers atteints, avaient les caractéristiques culturales que nous avons décrites avec quelques petites modifications dans un faible pourcentage de races. Ces caractéristiques sont les mêmes que celles trouvées par SenLemaN, Bishop-GLEY, dans une étude effectuée sur plus de mille races (Institut prussien de Recherches laitières de Kiel); par Klimmer, Haupt-Roots (Ecole vétérinaire de Leipzig); par DinRnhogger (Ecole vétérinaire de Vienne); par Menk (Institut d'Hygiène de Dantzig), et d'autres chercheurs.

Quelques-unes des variations des caractéristiques eulturales fondamentales décrites dans la présente étude ont été publiées pour quelques races dans des nombreuses études indiquées ei-dessus, et spécialement par L. J. Edwards, Jones et Seelemann. Nous avons observé des formes dissociées dans plusieurs de nos races de streptococcus mastitidis.

Le streptococcus mastitidis ou agalactiae est-il un streptocoque hémolytique ? - En raison de la bibliographie copieuse existant sur le streptococcus mastitidis, ou agalactiae, qui estime que son caractère non hémolytique est une de ses caractéristiques spéciales, nous estimons que les streptocoques hémolytiques trouvés quelquefois dans le mammite n'ont pas droit à la dénomination de streptococcus mastitidis. Des streptocoques hémolytiques ont été trouvés spécialement nombreux dans des cas de mammite par Jones. Carpenter a trouvé les hémolytiques formant $6 \%$ et les non hémolytiques $78,6 \%$. Minetr a trouvé $53 \%$ d'hémolytiques dans 113 cas de mammite.

Des manuels récents de bactériologie (LeHMAnN-NeUmanNBreED, Bactériologie déterminative, 1931, p. 226; BoECKer, KaUfMann, Robert KосH, Diagnostic bactériologique, 1931, p. 69 ; Hegan, Kolle-Wassermann, Klimmer, Krusse, etc.) indiquent que le streptococcus mastitidis est un streptocoque non hémolytique, et le décrivent comme un streptocoque alpha ou gamma-hémolytique. CarPenter, ayant trouvé que dans 76 troupeaux étudiés, 
$80 \%$ des échantillons étaient non hémolytiques et $6 \%$ hémolytiques, estime qu'il n'est pas convenable de placer sous le même nom spécifique deux organismes ayant une différence aussi sensible.

En dehors du test ordinaire sur plaque de sang en conditions - aérobiques et anaérobiques, nous avons utilisé le test d'hémolyse de $1 \mathrm{~cm}^{3}$ de culture de bouillon dans une suspension à $5 \%$ de globules rouges de mouton lavés et un autre test consistant à transférer un disque d'un centimètre de diamètre d'agar-sang ordinaire aux cultures de bouillon-sérum de 24 heures.

Sur plaques agar-sang étalées, des colonies profondes de streptocoques de la mammite sont souvent environnées par une zone de globules rouges incomplètement hémolysés, qui peuvent être occasionnellement considérés comme hémolytiques.

Je suis incliné à croire que dans beaucoup de eas de races de streptococcus agalactiae considérées comme hémolytiques, on peut avoir affaire à des races mélangées de deux sortes de streptocoques ou de streptococcus mastitidis avec des microcoques, ce qui se produit très souvent, ou bien les streptocoques étudiés sont des pyogènes ou d'autres streptocoques hémolytiques. J'ai plus souvent trouvé des streptocoques hémolytiques dans du lait commercial mélangé que dans du lait trait aseptiquement et nous avons isolé des streptocoques hémolytiques de toutes les parties voisines des vaches.

Le streptocoque non hémolytique décrit par HADLEY et FrosT sous le nom de streptococcus mitis et comme étant responsable de la plupart des cas de mammite, est exactement le même streptocoque qui a été trouvé responsable de la plus grande majorité des cas de mammite et reconnu dans la plupart des livres européens de bactériologie des cinq dernières années comme étant le streptococcus mastitidis ou streptococcus agalactiae et qui est aussi décrit sous ces noms dans les deux dernières éditions du Manuel de Bactériologie deseriptive de BERGEY, mais avec une description qui ne correspond pas aux vraies caractéristiques de ce streptocoque.

Si on n'étudiait le streptocoque que sur la base des réactions des sucres et de l'hémolyse, le streptococcus mastitidis pourrait être pris pour le streptococcus mitis ou même pour le fecalis ou le salivarius. Dans le plan d'Holman (1916), un streptocoque non hémolytique, positif pour le lactose et la salicine, négatif pour la mannite, est un streptococcus mitis. S'il est positif pour le lactose et la raffinose, négatif pour la salicine et la mannite, e'est un salivarius.

D'après la fermentation des sucres, le streptococcus mastitidis pourrait quelquefois être pris pour un mitis, quelquefois pour un fecalis, et même quelquefois comme un sativarius s'il fait fermenter le raffinose. Mais, si en même temps que la réaction des sucres, 
on utilise le lait tournesolé, le lait au bleu de méthylène, le $p \mathbf{H}$ final, la faculté de clivage de l'hippurate de sodium, on trouvera toujours qu'il a une individualité complètement distincte du mitis ou du fecalis, qui se montrent eux-mêmes nettement différents dans ces tests et aussi dans le développement à $20^{\circ}$ sur gélatine, dans la réduction du rouge au neutre, dans la température mortelle, dans son aspect morphologique et même dans l'aspect de sa colonie dans la grande liste des réactions des sucres, dans le final $p H$, et dans la détermination quantitative d'acide produit. Il est ainsi possible dans la plupart des cas de différencier suffisamment les streptococcus mastitidis ou agalactiae, du mitis, fecalis, salivarius, cremoris, et autres, rapprochés en apparence, et aussi des autres streptocoques d'origine animale et humaine.

Forme atypiques de streptococcus mastitidis. - Dans quelques autres cas, assez rares, $j$ 'ai trouvé des streptocoques avec des caractéristiques quelque peu différentes du type ordinaire de streptococcus mastitidis. Il n'est pas facile de savoir s'ils sont des formes dissociées, ou s'ils constituent des variétés séparées. Cette forme non typique de streptococcus mastitidis a donné des aspects culturaux ressemblant plus à ceux des groupes salivarius, mitis, lactis et fecalis. Ils sont dénommés par certains chercheurs formes non typiques de streptococcus mastitidis.

Je dois indiquer que les streptocoques de la mammite donnent très souvent lieu en sous-culture des formes dissociées et que les travaux faits avec quelques-unes de nos souches donnent lieu à laisser admettre que ce streptocóque peut se présenter sous une forme filtrable.

\section{PRÉVENTION ET TRAITEMENT}

Nous voudrions résumer nos expériences, sur ce point, faites surtout au Canada où des études systématiques nous ont été possibles avec les conclusions suivantes :

La propagation de la mammite peut être enrayée avec des mesures hygiéniques et prophylactiques, spécialement par la séparation des animaux malades, et par des mesures rigoureuses pour empêcher le passage de l'agent d'infection de ces animaux aux autres. Le plus important est d'éviter l'introduction des animaux malades dans les étables ou les troupeaux libres de maladie.

La vaccination préventive pratiquée en échelle avec répétition suffisante est, à notre avis, et selon notre expérience, une bonne mesure prophylactique.

De tous les traitements recommandés jusqu'à ce jour pour la mammite, pas un seul ne s'est montré ni 100 , ni $90 \%$ efficace. Mais avec une intense vaccination de 40 à $60 \mathrm{~cm}^{3}$ de vaccins streptrocciques 
et micrococciques frais mélangés avec 40 à $60 \mathrm{~cm}^{3}$ de lait et injectés trois à cinq fois avec cinq à sept jours d'intervalle et répétés, si nécessaire, deux à trois fois par année, nous avons obtenu des résultats bien manifestes, et dans la guérison et dans l'amélioration de la maladie.

\title{
LA CORROSION DES MÉTAUX PAR LE LAIT (1)
}

\author{
par \\ Colin G. FINK et Frederick A. ROHRMAN \\ de la Division d'Electrochimie de Columbia University (New-York).
}

\section{Introduction.}

Le lait est la plus importante des denrées alimentaires servant à la consommation de l'homme. Selon Houm [1], la production de lait aux Etats-Unis fut de 53.000.000 de tonnes en 1926, ayant une valeur marchande d'environ 4.000.000.000 de dollars. Lorsqu'on fait la comparaison avec d'autres industries, on constate que pour l'industrie automobile cette valeur atteint environ 3.500.000.000 de dollars, et pour l'industrie de l'acier, environ 3.000 .000 .000 de dollars.

Les dépôts coopératifs de lait, les usines de pasteurisation et le commerce ont exigé d'importantes installations d'appareils en métal. Une très importante partie du lait produit actuellement en Amérique est pasteurisée dans des appareils métalliques.

En contraste frappant avec son action, presque nulle, sur les organes du corps humain, l'effet du lait sur la plupart des métaux est très prononcé ; en fait, l'attaque des métaux par le lait est un des problèmes de corrosion les plus importants que devront résoudre la civilisation moderne et l'industrie.

\section{IMPORTANCE DE LA CORROSION PAR LE LAIT}

La corrosion des métaux par le lait ne devient pas seulement un problème économique à cause de son importance pour le renouvellement du matériel, mais également par la question de la valeur marchande des produits lactés et les facteurs physiologiques $q u$ 'elle influence. Un outillage défectueux peut être cause de la présence dans le lait de produits de la corrosion, dans des proportions telles, que, non seulement la saveur, mais également la valeur nutritive est altérée. La saveur peut être altérée uniquement par la solution de métal, d'où le goût métallique caractéristique, ou par l'action indirecte des métaux dissous sur les microorganismes du lait, ayant pour résultat la saveur « farineuse » [2].

(1) Journal of Dairy Science, vol. XV, $n^{\circ} 1$, janvier 1932, p. 73-86. 